\title{
FLAT PANEL PLANAR OPTIC DISPLAY
}

\author{
James T. Veligdan \\ Department of Advanced Technology \\ Brookhaven National Laboratory \\ Upton, NY 11973
}

November 1994

\section{DISCLAIMER}

This report was prepared as an account of work sponsored by an agency of the United States Government. Neither the United States Government nor any agency thereof, nor any of their employees, makes any warranty, express or implied, or assumes any legal liability or responsibility for the accuracy, completeness, or usefulness of any information, apparatus, product, or process disclosed, or represents that its use would not infringe privately owned rights. Reference herein to any specific commercial product, process, or service by trade name, trademark, manufacturer, or otherwise does not necessarily constitute or imply its endorsement, recommendation, or favoring by the United States Government or any agency thereof. The views and opinions of authors expressed herein do not necessarily state or reflect those of the United States Government or any agency thereof.

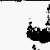




\section{DISCLAIMER}

Portions of this document may be illegible in electronic image products. Images are produced from the best available original document. 


\title{
Flat PANEL PLANAR OPTIC Display
}

\author{
James T. Veligdan \\ Brookhaven National Laboratory \\ Upton, NY 11973
}

\begin{abstract}
A prototype 10 inch flat panel Planar Optic Display, (POD), screen has been constructed and tested. This display screen is comprised of hundreds of planar optic glass sheets bonded together with a cladding layer between each sheet where each glass sheet represents a vertical line of resolution. The display is 9 inches wide by 5 inches high and approximately 1 inch thick. A 3 milliwatt $\mathrm{HeNe}$ laser is used as the illumination source and a vector scanning technique is employed.
\end{abstract}

\section{BACKGROUND}

For approximately 40 years, researchers have been searching for a flat panel TV screen. The president of the United States has identified Flat Panel Displays as a technology he sees as vital to the economic future of the Unites States. 1 From data communications in business, to entertainment on the family room TV, the Cathode Ray Tube (CRT) has proven itself to be indispensable as a means of displaying information. It ranks high in brightness, reliability, manufacturability and cost. However, its volume, weight, and power consumption have severely limited its portability which is required for the development of many new technologies. Future advancements of video display technologies will be dependent, to a large extent, on advances in flat panel displays.

Work on flat panel displays has been advancing on many fronts simultaneously with the largest fraction of research being conducted in the area of liquid crystal displays (active and passive.) Other popular technologies have been plasma displays, electro-luminescent devices, flat CRTs, and spatial light modulators. 
Although it has been known that lasers could provide an inherently high brightness and high resolution display, there has never been a method to accomplish this safely or compactly. Conventional laser projection, like the type employed at laser light shows, can provide a bright image on a flat screen in a tightly controlled environment where no one is able to intercept the laser beam with their eyes. Such a system would be unsafe to the eye and illegal in an uncontrolled home environment. A rear projection laser system could be used which could contain the dangerous levels of light, however, the physical size of such a device is no smaller than that of a conventional CRT. The Planar Optic Display, (POD), being described here uses neither front nor rear projection optics. It is an internal projection system which has the brightness of rear laser projection and the compactness of LCD screens.

\section{OPERATION THEORY}

[At the outset, it must be emphasized that the new technology presented here is the flat panel Planar Optic Display screen and not the use of a scanning laser beam to produce a video image. Therefore the lasers themselves and the laser scanner will receive only a basic discussion.l

Fiber optic wave guides have been well understood and used for decades. An internal fiber known as the core (refractive index $n$ ) is surrounded by a cladding (refractive index $<n$ ) so that light which enters the fiber within a known acceptance angle is confined within the fiber. This confinement occurs because of a process known as total internal reflection. The same process occurs if the internal core is a sheet of glass rather than a fiber of glass. It is understood that each internal core sheet must be adjacent to a sheet of cladding to ensure total internal reflection. Such a device, when constructed with many sheets or planes of glass is called a planar optic display. See Fig. 1. A planar optic device is analogous to a fiber optic device, however, there are a few very important distinctions. 
In a fiber optic, the angular information of the incident light beam is lost as the light exits the fiber. In a planar optic sheet, the angle of the incident light (in the plane of the sheet) is preserved at the exit of the sheet. This is a very important characteristic because one now has the capability to direct a laser beam into the entrance of a planar optic sheet and have the same scanning laser beam exit the sheet at a predetermined location. This is crucial to understanding the operation of this Planar Optic Display, (POD), flat panel screen.

Another difference between fiber optics and planar optics is the effect of diffraction on the propagating laser beam within the device. In a fiber optic the beam diameter is confined by the fiber and can never be larger than the fiber diameter. However, in the planar optic sheet, a propagating laser beam will continue to expand in one direction due to the effect of diffraction.

A single planar optic sheet is not very useful by itself, but if you were to stack 1000 different length sheets together, a new type of flat panel screen becomes evident as shown in Figure 1. For the purpose of this discussion, we consider a flat panel POD with a 2 meter diagonal display; 1 meter high by 1.33 meters wide. The panel contains 1,000 planar sheets, each 50 microns thick, for a total thickness of approximately $5 \mathrm{~cm}$. (A 6 tt. diagonal display, 2 inches thick)

\section{Vertical Resolution}

Figure 2 shows a detail of a section of the planar optic screen. The laser beam enters the planar optic display from the bottom. [For clarity, the laser beam diameter is shown to be much smaller than the planar core, but in reality the laser beam diameter should match the core diameter.] The cladding confines the laser within the core and the scanner rasters the laser beam along the length of the planar sheet. Each planar sheet corresponds to exactly one vertical line of resolution. Therefore, to attain a HDTV format with 1,000 lines of vertical resolution, the screen must contain 1,000 planar sheets. The laser light exits each planar sheet at the front frosted face which 
diffuses the beam to provide an extremely wide viewing angle, like conventional CRTs. This diffusive face is also makes the screen totally eye safe even if the scanner failed and the laser beam were fixed at a single spot.

\section{Horizontal Resolution}

Although the vertical resolution is determined by the number of planar sheets, the horizontal resolution can be limited by 2 factors; the modulation rate of the laser and the spreading effect of diffraction on the laser beam. As the diode laser beam is being scanned from left to right, its current must be modulated fast enough to produce 1,000 discrete pixels. Therefore to achieve a horizontal resolution of 1,000 for a high definition display, the laser diode must be turned on and off (or varied) 1,000 times during each horizontal scan. This high resolution would require the diode laser to be modulated at $1 \mathrm{MHz}$ (One million times per second). A conventional frame rate (30 frames per second) would require that the diode be modulated at $30 \mathrm{MHz}$. Therefore, the diode laser does not appear to be a limiting factor in attaining optimum horizontal resolution.

The effect of diffraction, however, will ultimately limit the attainable horizontal resolution. As the laser beam travels within the planar sheet, a 50 micron diameter laser beam would expand far too much to provide high resolution at the face of the screen 1 meter away. By expanding the laser beam to a diameter of 500 microns, the diffraction effect is now reduced so that the spot size at the screen surface is only $1 \mathrm{~mm}$. Since the screen is 1.33 meters wide, the horizontal resolution limit is 1330 . In order to obtain the optimum horizontal and vertical resolution simultaneously, a rectangular, rather than circular, beam profile is needed. Fortuitously, the diode laser, by its very nature, provides just such a beam profile. Thus, the ideal laser beam size for a 2 meter flat panel POD is 50 microns by 500 microns. 
A planar optic screen as shown in Figure 1 can be made as small or as large as desired, within the capability of manufacturers to produce the core material for the planar optics. For larger screens, one must use diodes with higher output powers or couple the output from several diodes. The laser scanner for a POD screen can be either mechanical, electro-optic, or acousto-optic depending on the need. For extremely high rate scanning applications, an active matrix liquid crystal display (AMLCD) can be placed on the input face for individual pixel control. Then all 1,000 horizontal lines could be scanned at once. For color applications, each of the three primary colors can be scanned sequentially to reduce the number of required LCD pixels.

Three dimensional TV (using light polarization) becomes a possibility with this Planar Optic Display since each planar optic sheet retains the polarization information of the incident laser beam. A second set of diode lasers with opposite polarization would be integrated with the scanner such that the two polarizations are time interlaced on the surface of the screen.

The question of manufacturing the planar optic screen should now be addressed. Although every POD made to date has used glass, a production screen should be plastic due to weight and cost considerations. After researching this subject it was discovered that the screen manufacturing problem has already been addressed by DOW Chemical Company. They have developed an inexpensive process to coextrude alternating layers of transparent PC/styreneacrylonitrile with alternating indices of refraction. This is exactly what is needed for a POD screen. Furthermore, they have managed to extrude the material so that the layers are as thin as 1 micron. An excellent description of the process is contained in the literature ${ }^{2}$. 


\section{EXPERIMENTAL RESULTS}

The initial 'proof of principle' experiment consisted of a $2.5 \mathrm{~cm}$ (1 in.) planar optic display where the individual glass wave guides were 75 microns thick. The glass sheets were bonded together with a low refractive index and low viscosity epoxy (\#328) manufactured by Epoxy Technology Inc. Although the resolution looked good (approximately 35 lines $/ \mathrm{cm}$ ), the contrast was poor because the display was quite opaque. We decided that future displays should be black to improve overall contrast. The next 15 displays were constructed using various epoxies and blackening agents in order to obtain maximum contrast. These displays were $10 \mathrm{~cm}(4 \mathrm{inch})$, diagonal, and used 200 micron thick glass, as we did not have the thinner glass in large sizes. The optimal display used lampblack (from Fisher Scientific) mixed with VA-6 epoxy (from Epoxy Technology Inc) as the bonding agent for the sheets of glass.

\section{The Prototype}

In order to make the $25 \mathrm{~cm}$ (10 inch) display, 145 sheets of glass (200 microns thick) were prepared as follows. The material was Schott D 263 borosilicate glass with a refractive index of 1.52. The sheets of glass were cut so that the first sheet was a full size sheet. This becomes the rear of the screen. Each successive sheet was cut 1 $\mathrm{mm}$ shorter and placed on top of the previous sheet. The last, and front-most sheet, is now only a thin strip of glass. The sheets were bonded together with the VA-6 epoxy (refractive index $=1.50$ ) and the front face was ground flat to act as the display surface. The bottom face of this stack of glass was ground and polished to act as the light input surface.

In order to test the Planar Optic Display, it was necessary to use a laser projection system. This system was built by Laser Images Inc. (Van Nuys, CA) and uses a $\mathrm{He} \mathrm{Ne}$ laser which emits 3 milliwatts of light. Vector scanning is accomplished through the use of low mass mirrors on high speed galvanometers. The animation software is called "Choreographics" and runs on a Macintosh computer. 


\section{Display Characteristics}

The diffusive nature of the screen allows for a very wide viewing angle, approximately 120 degrees. The brightness of the display has no inherent limit since the source laser controls the ultimate brightness. When the laser is off, the screen appears flat black. Because of this contrast, the colors appear brilliant. The measured resolution of this prototype display is 142 lines vertical and 400 lines horizontal. A photograph of the Planar Optic Display and its scanner is shown in Figure 3.

\section{Dimensional Viewing}

This display can be used for 3 dimensional (3D) viewing if a polarization algorithm is used and the viewer wears polarized glasses. The POD was found to maintain the polarization information of the laser beam, however a small amount of scattering of the polarization occurs at the diffusive display face. This will not have a deleterious effect on 3D operation since the amount of scattering was measured and found to be small; a crossed polarizer extinction ratio of 100 was measured.

\section{CONCLUSIONS}

The Planar Optic Display has been successfully demonstrated using vector scanning techniques running animation sequences. The display has high brightness, high contrast and an exceptionally wide viewing angle.

\section{ACKNOWLEDGMENTS}

I would like to thank Mr. Dwayne Branch for his skill in the cutting, handling and bonding of the microthin glass. Mr. Barry Lafler deserves much thanks for his help in the grinding and polishing of the display. I also wish to thank Mitch Hartman at Laser Images for providing the laser scanning system.

\section{REFERENCES}

1. Electronic Engineering Times, March 22, $1993 \quad$ Pg. 24

2. Journal of Plastic Film \& Sheeting, Vol. 4, 1988, Pages 104-115. 


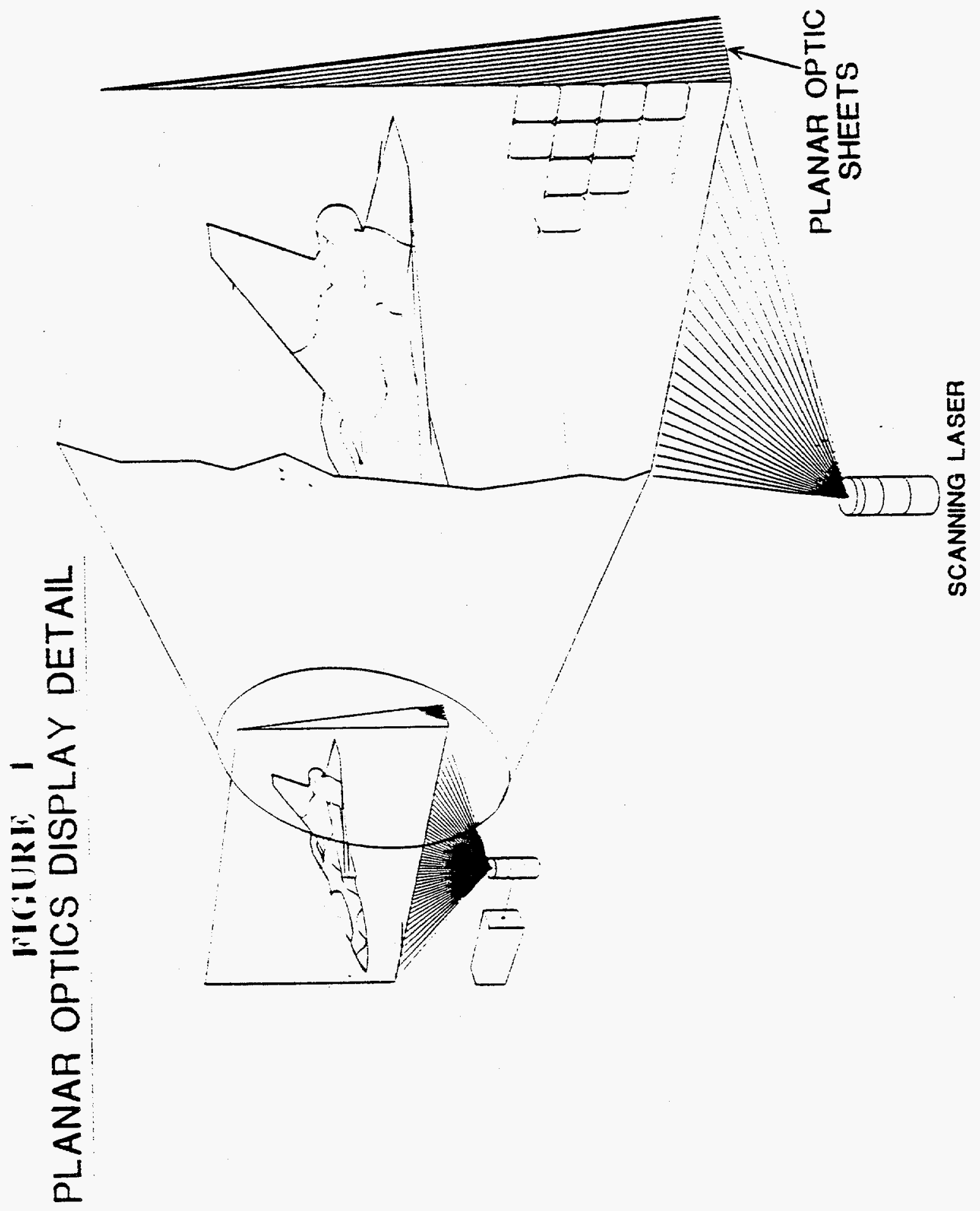




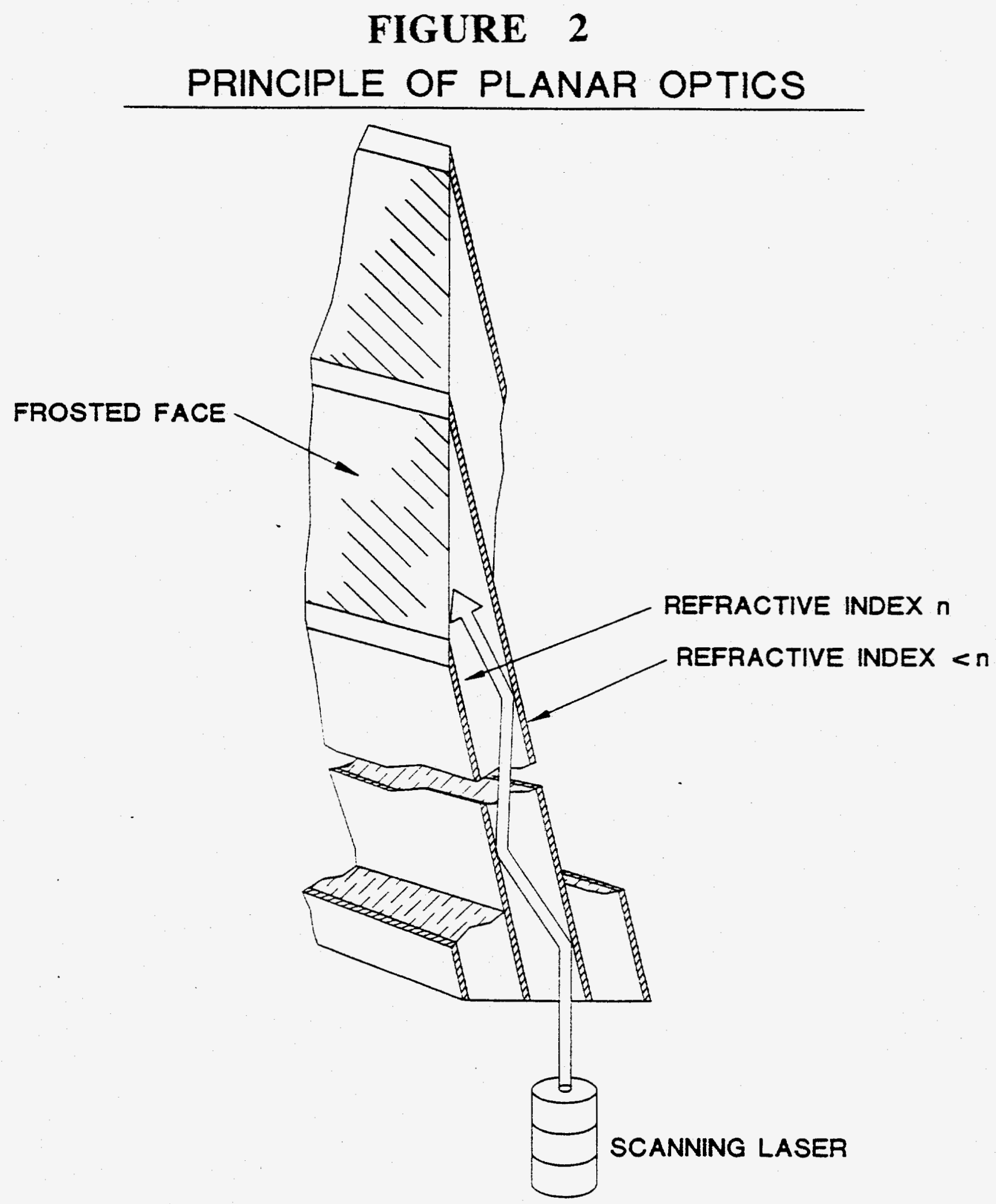




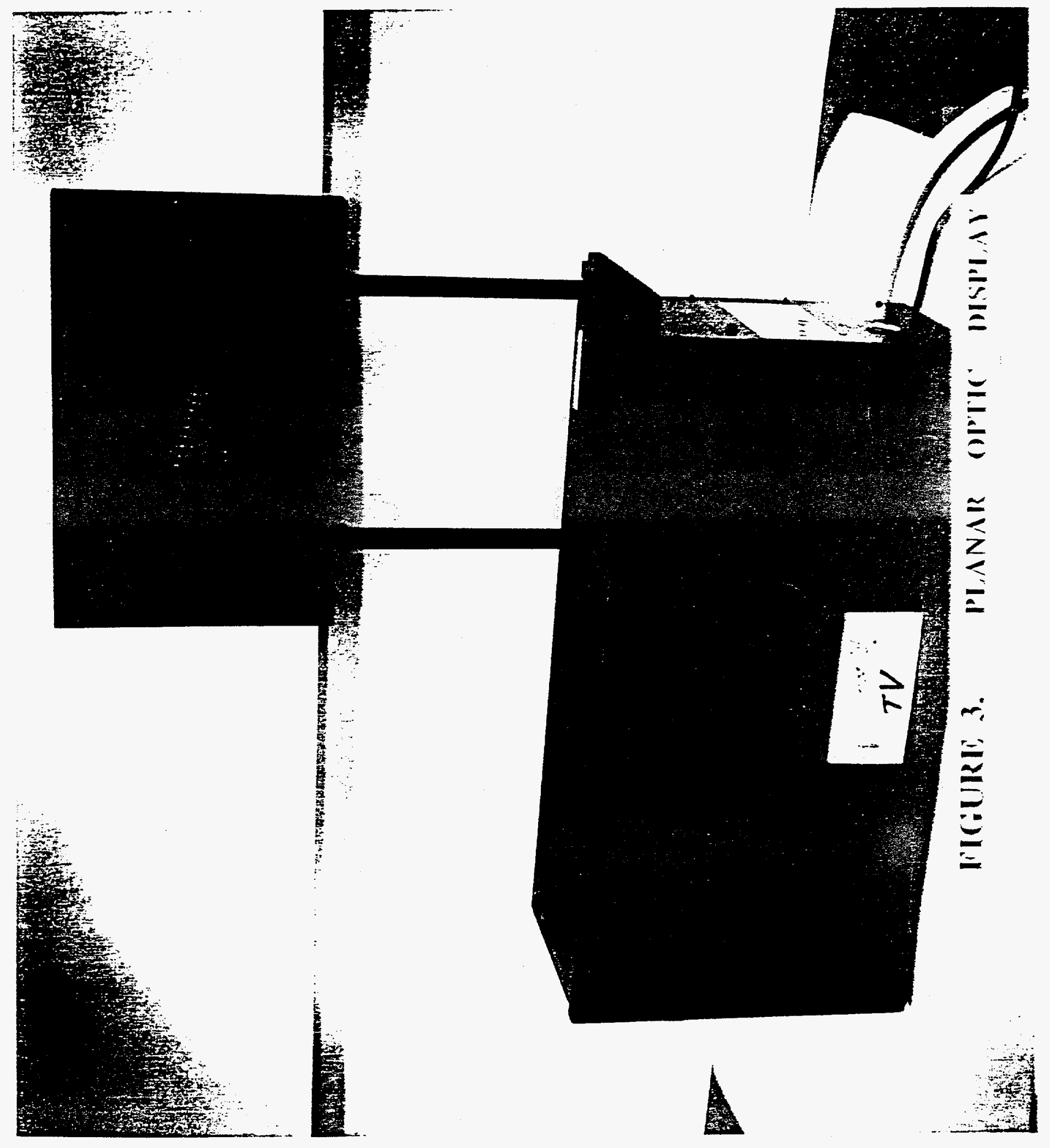




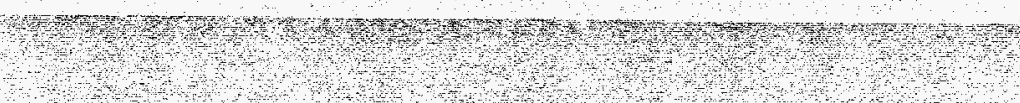
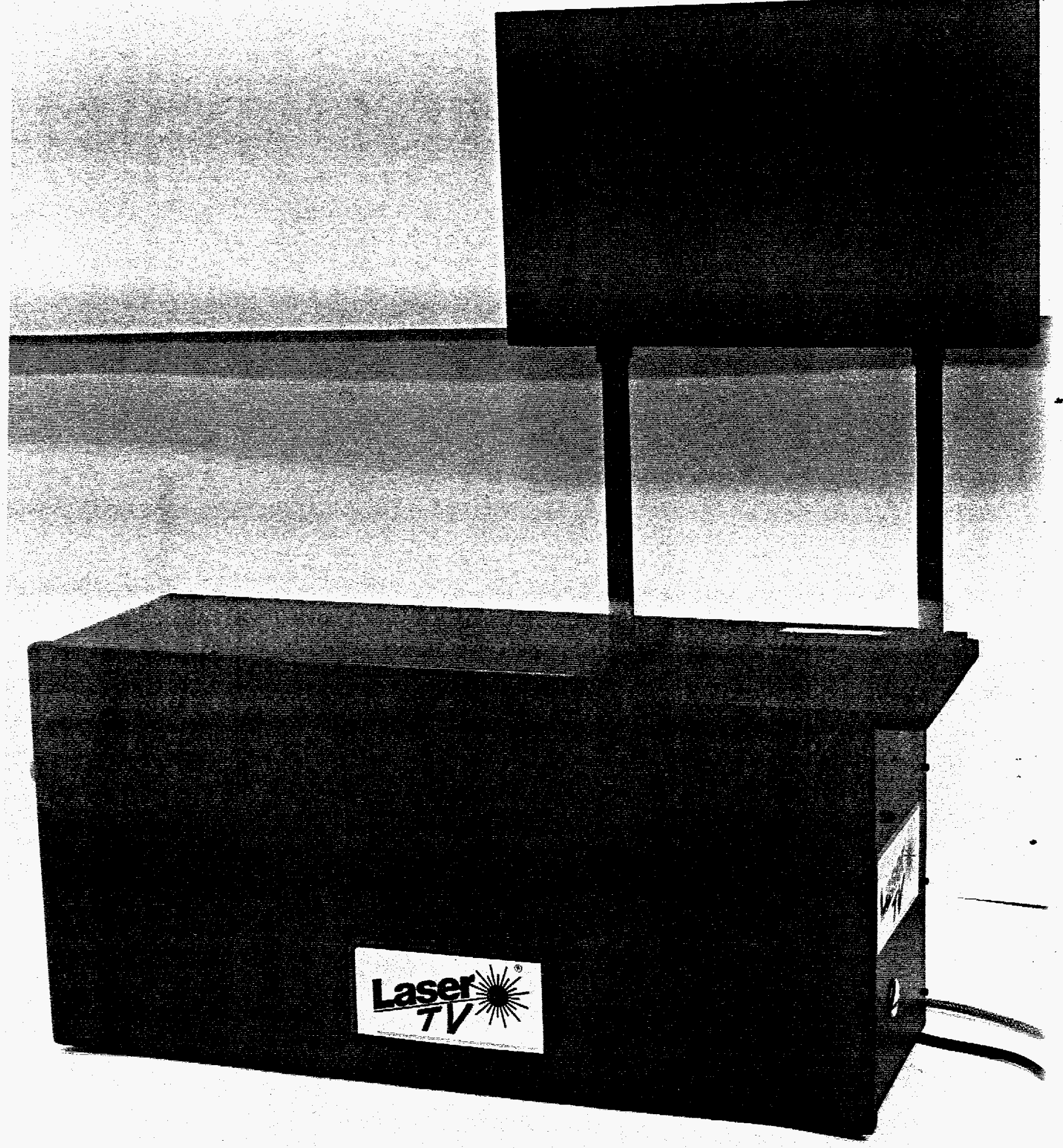